\title{
A class of global large, smooth solutions for the magnetohydrodynamics with the Hall and ion-slip effects
}

February 27, 2020

\author{
HUALI ZHANG* \\ Changsha University of Science and Technology \\ School of Mathematics and Statistics \\ Changsha, 410114, People's Republic of China.
}

\begin{abstract}
In this paper, the Cauchy's problem for fractional MHD system with the Hall and ion-slip effects is considered. By exploring the structure of semilinear and quasilinear terms, we prove the global existence of solutions for a class of large initial data. Both the velocity and magnetic fields could be arbitrarily large in $H^{3}\left(\mathbb{R}^{3}\right)$.
\end{abstract}

\section{Introduction}

In this paper we consider the following incompressible magnetohydrodynamics with the Hall and ion-slip effects:

$$
\left\{\begin{array}{l}
u_{t}+\nu \Lambda^{\alpha} u+u \cdot \nabla u+\nabla p-b \cdot \nabla b=0, \\
b_{t}+\mu \Lambda^{\beta} b+u \cdot \nabla b-b \cdot \nabla u+\sigma \nabla \times((\nabla \times b) \times b)-\kappa \nabla \times(((\nabla \times b) \times b) \times b)=0, \\
\nabla \cdot u=0, \quad \nabla \cdot b=0, \\
\left.u\right|_{t=0}=u_{0},\left.\quad b\right|_{t=0}=b_{0},
\end{array}\right.
$$

on the domain $(t, x) \in \mathbb{R}^{+} \times \mathbb{R}^{3}$, where $\Lambda=\sqrt{-\Delta}, \beta=2, \alpha \in[0,2]$. Here $u=\left(u_{1}, u_{2}, u_{3}\right)^{\mathrm{T}}, b=$ $\left(b_{1}, b_{2}, b_{3}\right)^{\mathrm{T}} \in \mathbb{R}^{3}$ denote the fluid velocity and magnetic fields respectively. The scalars $p, \nu, \mu$ are the pressure, viscosity, magnetic diffusivity respectively $(\nu, \mu$ are positive constants). $\kappa \geq 0, \sigma$ are constants. The Hall term $\nabla \times((\nabla \times b) \times b)$ is for the Hall effect, and $\nabla \times((\nabla \times b \times b) \times b)$ for ion-slip effect. $u_{0}$ and $b_{0}$ are the initial data satisfying

$$
\nabla \cdot u_{0}=\nabla \cdot h_{0}=0
$$

Equation 1.1 is important to describe some physical phenomena, e.g., in the magnetic reconnection in space plasmas, star formation, neutron stars and dynamo. In the case 
$\sigma=\kappa=0$, Equation 1.1 reduces to the standard MHD equations; in the case $\kappa=0$, Equation 1.1 reduces to Hall-MHD system. They has been extensively researched by a lot of excellent works, for instances [1]-[4], [6]-[11], [17]-[19] and [15, 28, 27, 35, 32].

For MHD system with the Hall and ion-slip effects, there are some interesting results related to the well-posedness theory, see references [23, 24, 25]. Recently, Fan et.al in [14] established global existence and time decay for small solutions. Very recently, Zhao and Zhu in [34] gave a proof of global existence for small solutions under weaker smallness conditions. However, none of results are known for MHD system with the Hall and ion-slip effects for general initial data without smallness conditions. It's quite rare to prove the existence of large, smooth, global solutions for quasilnear system. Under a class of large initial data, we found some results for incompressible Navier-Stokes equations and incompressible standard MHD equations, see [10, 9, 17, 19, 35, 22, 33, for details. Those motivate us to study the global well-posedness of Cauchy's problem of Equation 1.1 with large inital data. But the Hall and ion-slip term heightens the level of nonlinearity of the standard MHD system from a second-order semilinear to a second-order quasilinear level, significantly making its qualitative analysis more difficult. To the author's knowledge, it's quite rare to prove the existence of large, smooth, global solutions for quasilnear system. Based on the idea of LeiLin-Zhou in [17, we study the existence of global large solutions. It's not a trivial extension from Hall-MHD equations to Equation 1.1, for the additional difficulty of Equation 1.1 arise from: (1) the cubic quasi-linear term which requires some new cancellation estimates; (2) the proof of nonlinear smallness for some cross term. Using the large initial data constructed in [17 and combining nonlinear structures with commutator energy estimates, we are very fortunately to go through these difficulties - estimates (2.17)-(2.20) and (3.12).

The aim of this paper is to prove the existence of a unique, global smooth solution of MHD system with the Hall and ion-slip effects in $H^{3}\left(\mathbb{R}^{3}\right)$. Our result completely drops the smallness condition on the initial data.

Before we state our main results, we first give some notations. Let $\chi(x) \in C_{0}^{\infty}\left(\mathbb{R}^{3}\right)$ be a cut off function satisfying $|\chi(x)| \leq 2$ and

$$
\begin{aligned}
& \chi(x) \equiv 1, \quad \text { for }|x| \leq 1 ; \quad \chi(x) \equiv 0, \quad \text { for }|x| \geq 2, \\
& \left|\nabla^{k} \chi(x)\right| \leq 2, \quad 0 \leq k \leq 5 .
\end{aligned}
$$

Denote

$$
\chi_{M_{0}}(x):=\chi\left(\frac{x}{M_{0}}\right) .
$$

Here $M_{0}$ is a positive constant. Let $v_{0}$ be that constructed by Lei et al. [17, and it has the following properties

$$
\begin{aligned}
& \nabla \cdot v_{0}=0, \quad \nabla \times v_{0}=\sqrt{-\Delta} v_{0}, \\
& \operatorname{supp} \hat{v}_{0} \subseteq\{\xi|1-\delta \leq| \xi \mid \leq 1+\delta\}, \quad 0<\delta \leq \frac{1}{2}, \\
& \left\|\hat{v}_{0}\right\|_{L^{1}} \leq M_{1}, \quad\left|\nabla^{k} v_{0}\right| \leq \frac{M_{2}}{1+|x|}, \quad 0 \leq k \leq 5,
\end{aligned}
$$

where $M_{1}, M_{2}$ are positive constants, $\hat{v}_{0}$ is the Fourier transform of $v_{0}$ and the operator 
$\sqrt{-\Delta}$ is defined through the Fourier transform

$$
\widehat{\sqrt{-\Delta}} f(\xi)=|\xi| \hat{f}(\xi)
$$

Our main result is as follows.

Theorem 1.1. Consider Cauchy's problem 1.1]1.2. Suppose that

$$
\begin{aligned}
& u_{0}=u_{01}+\chi_{M_{0}} u_{02}, \\
& b_{0}=b_{01}+\chi_{M_{0}} b_{02} .
\end{aligned}
$$

with

$$
\begin{aligned}
& \nabla \cdot u_{02}=\nabla \cdot b_{02}=0, \\
& u_{02}=\alpha_{1} v_{0}, \quad b_{02}=\alpha_{2} v_{0},
\end{aligned}
$$

where $\chi_{M_{0}}, v_{0}$ are stated as above. $\alpha_{1}, \alpha_{2}$ are two real constants. Then there exist constants $\delta^{-\frac{1}{2}} \geq M_{0} \gg 1$ depending on $M_{1}, M_{2}, \alpha_{1}, \alpha_{2}, \mu, \nu, \eta$ such that Cauchy's problem 1.1 1.2 has a unique, global smooth solution provided that

$$
\left\|u_{01}\right\|_{H^{3}}+\left\|b_{01}\right\|_{H^{3}} \leq M_{0}^{-\frac{1}{2}} .
$$

Remark 1.1. For

$$
\begin{aligned}
\left\|u_{0}\right\|_{L^{\infty}}+\left\|b_{0}\right\|_{L^{\infty}} & \leq M_{1}, \\
\left\|u_{0}\right\|_{H^{3}}+\left\|b_{0}\right\|_{H^{3}} & \leq\left(M_{0}^{-\frac{1}{2}}+\left(\left|\alpha_{1}\right|+\left|\alpha_{2}\right|\right) \sum_{k=0}^{3} \frac{M_{2}}{M_{0}^{k}}\right),
\end{aligned}
$$

and the constant $M_{1}, M_{2}$ can be arbitrary large, thus our initial data can be arbitrary large. Comparing with 14, 34], our result can be seen as an non-trival improvement of Fan et al.'s and Zhao et al.'s work, for we completely drops the smallness condition on the initial data.

Remark 1.2. The parameter $\alpha, \beta$ indicates the strength of dissipation for velocity and magnetic field respectively. If the parameter $\alpha, \beta$ is larger, then the corresponding dissipation is stronger. When $\sigma=\kappa=0$, the conclusion in Theorem 1.1 still holds for all $\alpha, \beta \in[0,2]$. If $\sigma \neq 0$ or $\kappa \neq 0$, considering the quasilinear terms for magnetic field on (1.1), then the strong dissipative term $\mu \Delta b(\beta=2)$ may be necessary to compensate for the loss of regularity in exploring large solutions.

Remark 1.3. In the limiting case $\delta=0, \nabla \times u_{02}=u_{02}, \nabla \times b_{02}=b_{02}$, and the flow, magnetic field are called Beltrami flow and force-free fields respectively. Let us also mention that the magnetic energy achieves the minimum value for force-free fields, one can refer [30] for details.

Remark 1.4. We throughout use a notation $C$. It may be different from line to line, but it is a universal positive constant in this paper. 
The proof of Theorem 1.1 is based on a perturbation argument along with a standard cut-off technique, and the perturbation is as large as the initial data. Compared with HallMHD equations, a part of the nonlinearities may not be small for Equation 1.1 (see (3.3)). Fortunately, by combining the nonlinear structure of the term and commutator estimates, these terms can be estimated carefully.

This paper is organized as follows: In section 2, we introduce commutator estimates and give some estimate of quadratic and cubic terms. Section 3 is devoted to prove the global existence and uniqueness of large smooth solutions for Equation [1.1,

\section{Preliminaries}

Lemma 2.1. [3] Let $s>0$. Let $p, p_{2}, p_{3} \in(1, \infty)$ and $p_{2}, p_{4} \in[1, \infty)$ satisfy

$$
\frac{1}{p}=\frac{1}{p_{1}}+\frac{1}{p_{2}}=\frac{1}{p_{3}}+\frac{1}{p_{4}} .
$$

Then there exist two constants $C_{1}, C_{2}$,

$$
\begin{aligned}
& \left\|\Lambda^{s}(f g)\right\|_{L^{p}} \leq C_{1}\left(\left\|\Lambda^{s} f\right\|_{L^{p_{1}}}\|g\|_{L^{p_{2}}}+\left\|\Lambda^{s} g\right\|_{L^{p_{3}}}\|f\|_{L^{p_{4}}}\right), \\
& \left\|\left[\Lambda^{s}, f\right] g\right\|_{L^{p}} \leq C_{2}\left(\left\|\Lambda^{s} f\right\|_{L^{p_{1}}}\|g\|_{L^{p_{2}}}+\left\|\Lambda^{s-1} g\right\|_{L^{p_{3}}}\|\nabla f\|_{L^{p_{4}}}\right) .
\end{aligned}
$$

Let $f, g$ satisfy

$$
\left\{\begin{array}{l}
f_{t}+\nu \Lambda^{\alpha} f=0, \\
t=0: f=u_{02}
\end{array}\right.
$$

and

$$
\left\{\begin{array}{l}
g_{t}-\mu \Delta g=0, \\
t=0: g=b_{02} .
\end{array}\right.
$$

Therefore, we have

$$
f=e^{-\nu t \Lambda^{\alpha}} u_{02}, \quad g=e^{\mu t \Delta} b_{02} .
$$

Lemma 2.2. Let $f, g$ be defined in (2.1), (2.2). It holds

$$
\begin{aligned}
& \nabla \cdot f=0, \quad \nabla \times f=\sqrt{-\Delta} f, \\
& \nabla \cdot g=0, \quad \nabla \times g=\sqrt{-\Delta} g, \\
& \left|\nabla^{k} f\right| \leq \frac{\left|\alpha_{1}\right| M_{2}}{1+|x|} e^{-\frac{\nu t}{2 \alpha}}, \quad\left|\nabla^{k} g\right| \leq \frac{\left|\alpha_{2}\right| M_{2}}{1+|x|} e^{-\frac{\mu t}{4}}, \quad 0 \leq|k| \leq 5 .
\end{aligned}
$$

Proof. By

$$
\begin{aligned}
& \nabla \cdot v_{0}=0, \quad \nabla \times v_{0}=\sqrt{-\Delta} v_{0}, \\
& f=e^{-\nu t \Lambda^{\alpha}} u_{02}, \quad g=e^{\mu t \Delta} b_{02},
\end{aligned}
$$

we can deduce that

$$
\begin{array}{ll}
\nabla \cdot f=0, & \nabla \times f=\sqrt{-\Delta} f, \\
\nabla \cdot g=0, & \nabla \times g=\sqrt{-\Delta} g .
\end{array}
$$


We choose a $C^{\infty}\left(\mathbb{R}^{3}\right)$ cut-off function $\gamma(\xi)$ such that $\alpha \equiv 1$ on the support of $v_{0}$, and $\gamma(\xi) \equiv 0$ if $|\xi| \geq 1+2 \delta$ or $|\xi| \leq 1-2 \delta$. Then we have

$$
\begin{aligned}
& f(t, x)=\alpha_{1} e^{-\frac{\nu t}{2^{\alpha}}} \mathcal{F}^{-1}\left(e^{-\nu\left(|\xi|^{\alpha}-\frac{1}{2^{\alpha}}\right) t} \gamma(\xi)\right) * v_{0}, \\
& g(t, x)=\alpha_{2} e^{-\frac{\mu t}{2}} \mathcal{F}^{-1}\left(e^{-\mu\left(|\xi|^{2}-\frac{1}{2}\right) t} \gamma(\xi)\right) * v_{0} .
\end{aligned}
$$

In a result, we get

$$
\left|\nabla^{k} f\right| \leq \frac{\left|\alpha_{1}\right| M_{2}}{1+|x|} e^{-\frac{\nu t}{2 \alpha}}, \quad\left|\nabla^{k} g\right| \leq \frac{\left|\alpha_{2}\right| M_{2}}{1+|x|} e^{-\frac{\mu t}{4}}, \quad 0 \leq|k| \leq 5 .
$$

Lemma 2.3. Set $\tilde{f}:=\chi_{M_{0}} f, \tilde{g}:=\chi_{M_{0}} g$. Let $f, g, \chi_{M_{0}}$ be defined by (2.1), (2.2) and (1.4) respectively. Then we have

$$
\begin{gathered}
\|\tilde{f}\|_{W^{5, \infty}}+\|\tilde{g}\|_{W^{5, \infty}} \leq C\left(\left|\alpha_{1}\right| M_{1} e^{-\frac{\nu t}{2 \alpha}}+\left|\alpha_{2}\right| M_{1} e^{-\frac{\mu t}{4}}\right), \\
\|\tilde{f} \times(\nabla \times \tilde{f})\|_{H^{3}}+\|\tilde{g} \times(\nabla \times \tilde{g})\|_{H^{3}} \\
\leq C\left(\alpha_{1}^{2} e^{-\frac{\nu t}{2^{\alpha-1}}}+\alpha_{2}^{2} e^{-\frac{\mu t}{2}}\right)\left(\delta M_{0}^{\frac{3}{2}} M_{1}^{2}+M_{0}^{-1} M_{2}^{2}\right), \\
\|((\nabla \times \tilde{g}) \times \tilde{g}) \times \tilde{g}\|_{H^{3}} \leq C\left(\delta M_{0}^{\frac{3}{2}} M_{1}^{3}+M_{0}^{-1} M_{2}^{3}\right)\left|\alpha_{2}\right|^{3} e^{-\frac{3 \mu t}{4}}, \\
\int_{0}^{\infty}\|\tilde{f} \times \tilde{g}\|_{H^{3}}(t) d t \leq C M_{0}^{\frac{3}{2}} M_{1}^{2}(1+\alpha) \delta .
\end{gathered}
$$

Proof. Firstly, we have $\left|\nabla^{k} \chi_{M_{0}}\right| \leq C M_{0}^{-k}, k \leq 5$. Then

$$
\|\tilde{f}\|_{W^{5, \infty}}=\left\|\chi_{M_{0}} f\right\|_{W^{5, \infty}} \leq\left\|\chi_{M_{0}}\right\|_{W^{5, \infty}}\|f\|_{W^{5, \infty}} \leq C\|f\|_{W^{5, \infty}} .
$$

Using $\hat{f}=e^{-\nu|\xi|^{\alpha} t} \hat{u}_{02}$ and supp $\hat{u}_{02} \subseteq\{\xi|1-\delta \leq| \xi \mid \leq 1+\delta\}, 0<\delta \leq \frac{1}{2}$, we get

$$
\|f\|_{W^{5, \infty}} \leq\left\|(1+|\xi|)^{5} \hat{f}\right\|_{L_{\xi}^{1}} \leq C\left\|e^{-\nu t|\xi|^{\alpha}} \hat{u}_{02}\right\|_{L_{\xi}^{1}} \leq C\left|\alpha_{1}\right| M_{1} e^{-\frac{\nu t}{2 \alpha}}
$$

Similarly, we have

$$
\|g\|_{W^{5, \infty}} \leq C\left|\alpha_{2}\right| M_{1} e^{-\frac{\mu t}{4}}
$$

Adding (2.8) to (2.7), we obtain

$$
\|\tilde{f}\|_{W^{5, \infty}}+\|\left.\tilde{g}\right|_{W^{5, \infty}} \leq C\left(\left|\alpha_{1}\right| M_{1} e^{-\frac{\nu t}{2^{\alpha}}}+\left|\alpha_{2}\right| M_{1} e^{-\frac{\mu t}{4}}\right) .
$$

Secondly, we notice the fact

$$
\begin{aligned}
& \nabla \times\left(\chi_{M_{0}} f\right)=\nabla \chi_{M_{0}} \times f+\chi_{M_{0}} \nabla \times f, \\
& \nabla \times\left(\chi_{M_{0}} g\right)=\nabla \chi_{M_{0}} \times g+\chi_{M_{0}} \nabla \times g .
\end{aligned}
$$


Thus, we get

$$
\begin{aligned}
& \|\tilde{f} \times(\nabla \times \tilde{f})\|_{H^{3}}+\|\tilde{g} \times(\nabla \times \tilde{g})\|_{H^{3}} \\
= & \left\|\chi_{M_{0}} f \times\left(\nabla \times\left(\chi_{M_{0}} f\right)\right)\right\|_{H^{3}}+\left\|\chi_{M_{0}} g \times\left(\nabla \times\left(\chi_{M_{0}} g\right)\right)\right\|_{H^{3}} \\
\leq & C\left\|\chi_{M_{0}}^{2}\right\|_{H^{3}}\left(\|f \times(\nabla \times f)\|_{W^{3, \infty}}+\|g \times(\nabla \times g)\|_{W^{3, \infty}}\right) \\
& \quad+C\left\|\nabla\left(\chi_{M_{0}}^{2}\right)\right\|_{W^{3, \infty}}\left(\left|\left\|\left.f\right|^{2}\right\|_{H^{3}}+\left\|\left.|| g\right|^{2}\right\|_{H^{3}}\right)\right.
\end{aligned}
$$

and

$$
\begin{aligned}
& \|((\nabla \times \tilde{g}) \times \tilde{g}) \times \tilde{g}\|_{H^{3}} \\
= & \left\|\left(\left(\nabla \times\left(\chi_{M_{0}} g\right)\right) \times\left(\chi_{M_{0}} g\right)\right) \times\left(\chi_{M_{0}} g\right)\right\|_{H^{3}} \\
\leq & C\left(\left\|\chi_{M_{0}}^{3}\right\|_{H^{3}}\|((\nabla \times g) \times g) \times g\|_{W^{3, \infty}}+\left\|\nabla \chi_{M_{0}}^{3}\right\|_{W^{3, \infty}}\left\|g^{3}\right\|_{H^{3}}\right) .
\end{aligned}
$$

We calculate that

$$
\begin{aligned}
& \left\|\chi_{M_{0}}^{2}\right\|_{H^{3}}+\left\|\chi_{M_{0}}^{3}\right\|_{H^{3}} \leq C \sum_{i=0}^{3} M_{0}^{-i} M_{0}^{\frac{3}{2}} \leq C M_{0}^{\frac{3}{2}}, \\
& \left\|\nabla\left(\chi_{M_{0}}^{2}\right)\right\|_{W^{3, \infty}}+\left\|\nabla\left(\chi_{M_{0}}^{3}\right)\right\|_{W^{3, \infty}} \leq C \sum_{i=0}^{3} M_{0}^{-i-1} \leq C M_{0}^{-1} .
\end{aligned}
$$

For $f \times f=0, g \times g=0$, then we have

$$
\begin{aligned}
& \|f \times(\nabla \times f)\|_{W^{3, \infty}}+\|g \times(\nabla \times g)\|_{W^{3, \infty}} \\
= & \|f \times(\nabla \times f-f)\|_{W^{3, \infty}}+\|g \times(\nabla \times g-g)\|_{W^{3, \infty}} \\
\leq & \|f\|_{W^{3, \infty}}\|\nabla \times f-f\|_{W^{3, \infty}}+\|g\|_{W^{3, \infty}}\|\nabla \times g-g\|_{W^{3, \infty}} \\
\leq & C\left(\left\|(1+|\xi|)^{3} \hat{f}||_{L_{\xi}^{1}}\right\|(1+|\xi|)^{3}(|\xi|-1) \hat{f} \|_{L_{\xi}^{1}}\right) \\
& \quad+C\left\|(1+|\xi|)^{3} \hat{g}||_{L_{\xi}^{1}}\right\|(1+|\xi|)^{3}(|\xi|-1) \hat{g} \|_{L_{\xi}^{1}} \\
& \leq C M_{1}^{2} \delta\left(\alpha_{1}^{2} e^{-\frac{\nu}{2^{\alpha-1}} t}+\alpha_{2}^{2} e^{-\frac{\mu t}{2}}\right),
\end{aligned}
$$

and

$$
\begin{aligned}
\|((\nabla \times g) \times g) \times g\|_{W^{3, \infty}} & \leq\|\nabla \times g-g\|_{W^{3, \infty}}\|g\|_{W^{3, \infty}}^{2} \\
& \leq C \delta \|\left.\hat{g}(\xi)\right|_{L_{\xi}^{1}} ^{3} \\
& \leq C \delta M_{1}^{3}\left|\alpha_{2}\right|^{3} e^{-\frac{3 \mu t}{4}} .
\end{aligned}
$$

Noticing that supp $\widehat{|f|^{2}}$, supp $\widehat{|g|^{2}} \subseteq\{\xi|| \xi \mid \leq 2+2 \delta\}, 0<\delta \leq \frac{1}{2}$, we derive that

$$
\begin{aligned}
\left\||f|^{2}\right\|_{H^{3}}+\left\|\left.|| g\right|^{2}\right\|_{H^{3}} & \leq C\left|\left\|\left.f\right|^{2}\right\|_{L^{2}}+\right|\left\|\left.g\right|^{2}\right\|_{L^{2}} \\
& \leq C\left(\|f\|_{L^{4}}^{2}+\|g\|_{L^{4}}^{2}\right) \\
& \leq C\left(\alpha_{1}^{2} e^{-\frac{\nu t}{2^{\alpha-1}}} M_{2}^{2}+\alpha_{2}^{2} e^{-\frac{\mu t}{2}} M_{2}^{2}\right),
\end{aligned}
$$

and

$$
|||g|^{3}\left\|_{H^{3}} \leq C|||g|^{3}\right\|_{L^{2}} \leq C|| g \|_{L^{6}}^{3} \leq C\left|\alpha_{2}\right|^{3} M_{2}^{3} e^{-\frac{3 \mu t}{4}}
$$


Combining inequalities (2.10), (2.11) and (2.13), we get

$$
\|\tilde{f} \times(\nabla \times \tilde{f})\|_{H^{3}}+\|\tilde{g} \times(\nabla \times \tilde{g})\|_{H^{3}} \leq C\left(\alpha_{1}^{2} e^{-\frac{\nu t}{2^{\alpha-1}}}+\alpha_{2}^{2} e^{-\frac{\mu t}{2}}\right)\left(\delta M_{0}^{\frac{3}{2}} M_{1}^{2}+M_{0}^{-1} M_{2}^{2}\right) .
$$

Combining inequalities (2.9), (2.12) and (2.14), we deduce that

$$
\|((\nabla \times \tilde{g}) \times \tilde{g}) \times \tilde{g}\|_{H^{3}} \leq C\left(\delta M_{0}^{\frac{3}{2}} M_{1}^{3}+M_{0}^{-1} M_{2}^{3}\right)\left|\alpha_{2}\right|^{3} e^{-\frac{3 \mu t}{4}} .
$$

In what follows, we will estimate $\int_{0}^{t}\|\tilde{f} \times \tilde{g}\|_{H^{3}}(\tau) d \tau$. On one hand,

$$
\|\tilde{f} \times \tilde{g}\|_{H^{3}}=\left\|\chi_{M_{0}} f \times\left(\chi_{M_{0}} g\right)\right\|_{H^{3}} \leq\left\|\chi_{M_{0}}^{2}\right\|_{H^{3}}\|f \times g\|_{W^{3, \infty}} .
$$

On the other hand, supp $\widehat{f \times g} \subseteq\{\xi|| \xi \mid \leq 2+2 \delta\}, 0<\delta \leq \frac{1}{2}$. Then we have

$$
\|\tilde{f} \times \tilde{g}\|_{H^{3}} \leq C M_{0}^{\frac{3}{2}}\|\widehat{f \times g}\|_{L_{\xi}^{1}} .
$$

Calculate

$$
\begin{aligned}
\widehat{f \times g} & =\alpha_{1} \alpha_{2} \int_{\mathbb{R}^{3}} e^{-\nu|\xi-\eta|^{\alpha} t} \hat{v}_{0}(\xi-\eta) \times e^{-\mu|\eta|^{2} t} \hat{v}_{0}(\eta) d \eta \\
& =\frac{1}{2} \alpha_{1} \alpha_{2} \int_{\mathbb{R}^{3}}\left(e^{-\left(\nu|\xi-\eta|^{\alpha}+\mu|\eta|^{2}\right) t}-e^{-\left(\mu|\xi-\eta|^{2}+\nu|\eta|^{\alpha}\right) t}\right) \hat{v}_{0}(\xi-\eta) \times \hat{v}_{0}(\eta) d \eta
\end{aligned}
$$

and

$$
\begin{aligned}
& \left|e^{-\left(\nu|\xi-\eta|^{\alpha}+\mu|\eta|^{2}\right) t}-e^{-\left(\mu|\xi-\eta|^{2}+\nu|\eta|^{\alpha}\right) t}\right| \\
= & e^{-\nu|\xi-\eta|^{\alpha} t}\left|e^{-\mu|\eta|^{2} t}-e^{-\mu|\xi-\eta|^{2} t}\right|+e^{-\mu|\xi-\eta|^{2} t}\left|e^{-\nu|\xi-\eta|^{\alpha} t}-e^{-\nu|\eta|^{\alpha} t}\right| \\
\leq & C t e^{-\nu|\xi-\eta|^{\alpha} t}|| \xi-\left.\eta\right|^{2}-|\eta|^{2}\left|+C t e^{-\mu|\xi-\eta|^{2} t}\right||\xi-\eta|^{\alpha}-|\eta|^{\alpha} \mid \\
\leq & C e^{-\frac{\nu}{2}|\xi-\eta|^{\alpha}} \frac{|| \xi-\left.\eta\right|^{2}-|\eta|^{2} \mid}{|\xi-\eta|^{\alpha}}+C e^{-\frac{\mu}{2}|\xi-\eta|^{2} t} \frac{|| \xi-\left.\eta\right|^{\alpha}-|\eta|^{\alpha} \mid}{|\xi-\eta|^{2}} .
\end{aligned}
$$

In the support of $\hat{v}_{0}(\xi-\eta) \times \hat{v_{0}}(\eta)$, we have

$$
\frac{\| \xi-\left.\eta\right|^{2}-|\eta|^{2} \mid}{|\xi-\eta|^{\alpha}} \leq 3^{1-\alpha} \delta, \quad \frac{\| \xi-\left.\eta\right|^{\alpha}-|\eta|^{\alpha} \mid}{|\xi-\eta|^{2}} \leq 8 \alpha \delta .
$$

Therefore, we conclude that

$$
\int_{0}^{\infty}\|\tilde{f} \times \tilde{g}\|_{H^{3}}(t) d t \leq C M_{0}^{\frac{3}{2}} M_{1}^{2}(1+\alpha) \delta
$$

Then we complete the proof of Lemma 2.3 ,

\section{The proof of Theorem 1.1}

In this section, we will prove Theorem 1.1 using a perturbation argument along with a standard cut-off technique. 
Proof of Theorem 1.1. Let $\tilde{f}=\chi_{M_{0}} f, \tilde{g}=\chi_{M_{0}} g$, and $u=U+\tilde{f}, b=B+\tilde{g}$. Then $U, B$ satisfy

$$
\begin{gathered}
U_{t}+\nu \Lambda^{\alpha} U+\nabla\left(p+\frac{1}{2}|\tilde{f}|^{2}-\frac{1}{2}|\tilde{g}|^{2}\right) \\
=-U \cdot \nabla U-\tilde{f} \cdot \nabla U-U \cdot \nabla \tilde{f}+B \cdot \nabla B \\
+\tilde{g} \cdot \nabla B+B \cdot \nabla \tilde{g}+F, \\
\left.B_{t}-\mu \Delta B-\nabla \times((\nabla \times B) \times B) \times B\right) \\
=-U \cdot \nabla B-\tilde{f} \cdot \nabla B-U \cdot \nabla \tilde{g}+B \cdot \nabla U+\tilde{g} \cdot \nabla U+B \cdot \nabla \tilde{f} \\
-\eta \nabla \times((\nabla \times B) \times B)-\eta \nabla \times((\nabla \times B) \times \tilde{g}) \\
-\eta \nabla \times((\nabla \times \tilde{g}) \times B)-\eta \nabla \times((\nabla \times \tilde{g}) \times \tilde{g})+G \\
+\nabla \times(((\nabla \times B) \times B) \times \tilde{g})+\nabla \times(((\nabla \times B) \times \tilde{g}) \times B) \\
+\nabla \times(((\nabla \times B) \times \tilde{g}) \times \tilde{g})+\nabla \times(((\nabla \times \tilde{g}) \times B) \times B) \\
+\nabla \times(((\nabla \times \tilde{g}) \times B) \times \tilde{g})+\nabla \times(((\nabla \times \tilde{g}) \times \tilde{g}) \times B),
\end{gathered}
$$

where

$$
\begin{aligned}
F:=\tilde{f} & \times(\nabla \times \tilde{f})-\tilde{g} \times(\nabla \times \tilde{g})-\nu \Delta \chi_{M_{0}} f+2 \nu \nabla \cdot\left(\nabla \chi_{M_{0}} f\right), \\
G:=\nabla & \times(\tilde{f} \times \tilde{g})-\mu \Delta \chi_{M_{0}} g+2 \mu \nabla \cdot\left(\nabla \chi_{M_{0}} g\right)+\frac{1}{2} f \cdot \nabla \chi_{M_{0}}{ }^{2} g \\
& \quad-\frac{1}{2} g \cdot \nabla \chi_{M_{0}}{ }^{2} f-\nabla \times(((\nabla \times \tilde{g}) \times \tilde{g})-\nabla \times(((\nabla \times \tilde{g}) \times \tilde{g}) \times \tilde{g}) .
\end{aligned}
$$

In what follows, we will derive some energy estimates of $U$ and $B$.

\section{Step 1: Energy inequalities of $B$.}

Taking the derivatives $\Lambda^{k}, 0 \leq k \leq 3$ on Equation 3.2 and $L^{2}$ inner product with $\Lambda^{k} B$, we get

$$
\begin{aligned}
& \frac{1}{2} \frac{d}{d t}\|B\|_{H^{3}}^{2}+\|\nabla B\|_{H^{3}}^{2} \\
=I_{1} & +I_{2}+I_{3}+I_{4}+I_{5}+I_{6} \\
& \quad+J_{1}+J_{2}+J_{3}+\int_{\mathbb{R}^{3}} G \cdot B d x \\
& +K_{0}+K_{1}+K_{2}+K_{3}+K_{4}+K_{5}+K_{6},
\end{aligned}
$$

where

$$
\begin{aligned}
& I_{1}=-\sum_{0 \leq k \leq 3} \int_{\mathbb{R}^{3}} \Lambda^{k}(U \cdot \nabla B) \cdot \Lambda^{k} B d x, \quad I_{2}=-\sum_{0 \leq k \leq 3} \int_{\mathbb{R}^{3}} \Lambda^{k}(\tilde{f} \cdot \nabla B) \cdot \Lambda^{k} B d x \\
& I_{3}=-\sum_{0 \leq k \leq 3} \int_{\mathbb{R}^{3}} \Lambda^{k}(U \cdot \nabla \tilde{g}) \cdot \Lambda^{k} B d x, \quad I_{4}=\sum_{0 \leq k \leq 3} \int_{\mathbb{R}^{3}} \Lambda^{k}(B \cdot \nabla U) \cdot \Lambda^{k} B d x \\
& I_{5}=\sum_{0 \leq k \leq 3} \int_{\mathbb{R}^{3}} \Lambda^{k}(\tilde{g} \cdot \nabla U) \cdot \Lambda^{k} B d x, \quad I_{6}=\sum_{0 \leq k \leq 3} \int_{\mathbb{R}^{3}} \Lambda^{k}(B \cdot \nabla \tilde{f}) \cdot \Lambda^{k} B d x,
\end{aligned}
$$




$$
\begin{gathered}
J_{1}=-\sigma \sum_{0 \leq k \leq 3} \int_{\mathbb{R}^{3}} \Lambda^{k}((\nabla \times B) \times B) \cdot\left(\nabla \times \Lambda^{k} B\right) d x, \\
J_{2}=-\sigma \sum_{0 \leq k \leq 3} \int_{\mathbb{R}^{3}} \Lambda^{k}((\nabla \times B) \times \tilde{g}) \cdot\left(\nabla \times \Lambda^{k} B\right) d x, \\
J_{3}=-\sigma \sum_{0 \leq k \leq 3} \int_{\mathbb{R}^{3}} \Lambda^{k}((\nabla \times \tilde{g}) \times B) \cdot\left(\nabla \times \Lambda^{k} B\right) d x, \\
K_{0}=\kappa \sum_{0 \leq k \leq 3} \int_{\mathbb{R}^{3}} \Lambda^{k}(((\nabla \times B) \times B) \times B) \cdot\left(\nabla \times \Lambda^{k} B\right) d x, \\
K_{1}=\kappa \sum_{0 \leq k \leq 3} \int_{\mathbb{R}^{3}} \Lambda^{k}(((\nabla \times B) \times B) \times \tilde{g}) \cdot\left(\nabla \times \Lambda^{k} B\right) d x, \\
K_{2}=\kappa \sum_{0 \leq k \leq 3} \int_{\mathbb{R}^{3}} \Lambda^{k}(((\nabla \times B) \times \tilde{g}) \times B) \cdot\left(\nabla \times \Lambda^{k} B\right) d x, \\
K_{3}=\kappa \sum_{0 \leq k \leq 3} \int_{\mathbb{R}^{3}} \Lambda^{k}(((\nabla \times B) \times \tilde{g}) \times \tilde{g}) \cdot\left(\nabla \times \Lambda^{k} B\right) d x, \\
K_{4}=\kappa \sum_{0 \leq k \leq 3} \int_{\mathbb{R}^{3}} \Lambda^{k}(((\nabla \times \tilde{g}) \times B) \times \tilde{g}) \cdot\left(\nabla \times \Lambda^{k} B\right) d x, \\
K_{5}=\kappa \sum_{0 \leq k \leq 3} \int_{\mathbb{R}^{3}} \Lambda^{k}(((\nabla \times \tilde{g}) \times B) \times B) \cdot\left(\nabla \times \Lambda^{k} B\right) d x, \\
K_{6}=\kappa \sum_{0 \leq k \leq 3} \int_{\mathbb{R}^{3}} \Lambda^{k}(((\nabla \times \tilde{g}) \times \tilde{g}) \times B) \cdot\left(\nabla \times \Lambda^{k} B\right) d x .
\end{gathered}
$$

Firstly, we estimate $I_{1}, I_{2}$ in the following:

$$
\begin{aligned}
& \quad\left|I_{1}+I_{2}\right| \\
& \leq \sum_{0 \leq k \leq 3} \mid\left(\int_{\mathbb{R}^{3}}\left(\Lambda^{k}(U \cdot \nabla B)-\left(U \cdot \nabla \Lambda^{k} B\right) \cdot \Lambda^{k} B d x+\int_{\mathbb{R}^{3}}\left(\Lambda^{k}\left(\tilde{f} \cdot \nabla B-\tilde{f} \cdot \nabla \Lambda^{k} B\right)\right) \cdot \Lambda^{k} B d x\right) \mid\right. \\
& \quad+\left|\sum_{0 \leq k \leq 3} \int_{\mathbb{R}^{3}}\left(u \cdot \nabla \Lambda^{k} B\right) \cdot \Lambda^{k} B d x\right| \\
& \leq \sum_{0 \leq k \leq 3}\left(\mid \int_{\mathbb{R}^{3}}\left(\Lambda^{k}(U \cdot \nabla B)-\left(U \cdot \nabla \Lambda^{k} B\right) \cdot \Lambda^{k} B d x|+| \int_{\mathbb{R}^{3}}\left(\Lambda^{k}\left(\tilde{f} \cdot \nabla B-\tilde{f} \cdot \nabla \Lambda^{k} B\right)\right) \cdot \Lambda^{k} B d x \mid\right)\right. \\
& \leq C\left(\|\nabla U\|_{L^{\infty}}\|\nabla B\|_{H^{2}}+\|\nabla B\|_{L^{\frac{6}{\alpha}}}|| U \|_{W^{3, \frac{6}{2-\alpha}}}\right)\|B\|_{H^{3}} \\
& \quad+C\left(\|\nabla B\|_{H^{2}}\|\nabla \tilde{f}\|_{L^{\infty}}+\|\nabla B\|_{L^{6}}\|\tilde{f}\|_{W^{3,3}}\right)\|B\|_{H^{3}} .
\end{aligned}
$$

By Sobolev's inequality, we deduce that

$$
\left|I_{1}+I_{2}\right| \leq C\left(\left\|\Lambda^{\frac{\alpha}{2}} U\right\|_{H^{3}}|| \nabla B\left\|_{H^{3}}\right\| B\left\|_{H^{3}}+\right\| B \|_{H^{3}}^{2}\left(\|\tilde{f}\|_{W^{1, \infty}}+\|\tilde{f}\|_{W^{3,3}}\right)\right) .
$$

For $I_{3}$, it's easy for us to get

$$
\left|I_{3}\right| \leq C|| U\left\|_{H^{3}}|| B\right\|_{H^{3}}|| \tilde{g} \|_{W^{4, \infty}}
$$


For $I_{3}, I_{4}$, we derive that

$$
\begin{aligned}
\left|I_{4}+I_{5}\right| \leq C & \left(\|\nabla U\|_{H^{2}}\|\nabla B\|_{L^{\infty}}+\|\nabla U\|_{L^{3}}\|B\|_{W^{3,6}}\right)\|B\|_{H^{3}} \\
& +C\left(\|\nabla U\|_{H^{2}}\|\nabla \tilde{f}\|_{L^{\infty}}+\|\nabla U\|_{L^{6}}\|\tilde{f}\|_{W^{3,3}}\right)\|B\|_{H^{3}} \\
\leq & C\left(\left\|\Lambda^{\frac{\alpha}{2}} U\right\|_{H^{3}}\|\nabla B\|_{H^{3}}\|B\|_{H^{3}}+\|U\|_{H^{3}}\|B\|_{H^{3}}\left(\|\tilde{f}\|_{W^{1, \infty}}+\|\tilde{f}\|_{W^{3,3}}\right)\right) .
\end{aligned}
$$

Considering $I_{6}$, we have

$$
\left|I_{6}\right| \leq C|| B \|\left._{H^{3}}^{2}|| \tilde{f}\right|_{W^{4, \infty}} .
$$

Next step, we will estimate $J_{1}, J_{2}, J_{3}$. For

$$
\begin{aligned}
J_{1} & =\sum_{0 \leq k \leq 3} \sigma \int_{\mathbb{R}^{3}} \Lambda^{k}(\nabla \times B) \cdot \Lambda^{k}((\nabla \times B) \times B) d x \\
& =\sigma \sum_{0 \leq k \leq 3} \int_{\mathbb{R}^{3}}\left\{\Lambda^{k}((\nabla \times B) \times B)-\Lambda^{k}(\nabla \times B) \times B\right\} \cdot \Lambda^{k}(\nabla \times B) d x .
\end{aligned}
$$

Using Lemma 2.1, we deduce that

$$
\begin{aligned}
\left|J_{1}\right| & \leq C \eta \sum_{0 \leq k \leq 3}\left\|\Lambda^{k}(\nabla \times B)\right\|_{L^{2}}\left\|\Lambda^{k}((\nabla \times B) \times B)-\Lambda^{k}(\nabla \times B) \times B\right\|_{L^{2}} \\
& \leq C \eta\|\nabla B\|_{H^{3}}\left(\|\nabla \times B\|_{H^{2}}\|\nabla B\|_{L^{\infty}}+\|\nabla \times B\|_{L^{\infty}}\|B\|_{H^{3}}\right) \\
& \leq C \eta\|\nabla B\|_{H^{3}}^{2}\|B\|_{H^{3}} .
\end{aligned}
$$

For $J_{2}$, we calculate

$$
\begin{aligned}
J_{2}= & \sum_{0 \leq k \leq 3} \sigma \int_{\mathbb{R}^{3}} \Lambda^{k}(\nabla \times B) \cdot \Lambda^{k}((\nabla \times B) \times \tilde{g}) d x \\
= & \sigma \sum_{0 \leq k \leq 3} \int_{\mathbb{R}^{3}} \Lambda^{k}(\nabla \times B) \cdot\left(\Lambda^{k}((\nabla \times B) \times \tilde{g})-\Lambda^{k}(\nabla \times B) \times \tilde{g}\right) d x \\
& \quad+\sigma \sum_{0 \leq k \leq 3} \int_{\mathbb{R}^{3}}\left(\nabla \times \Lambda^{k} B\right) \cdot\left(\left(\nabla \times \Lambda^{k} B\right) \times \tilde{g}\right) d x \\
= & \sigma \sum_{0 \leq k \leq 3} \int_{\mathbb{R}^{3}} \Lambda^{k}(\nabla \times B) \cdot\left(\Lambda^{k}((\nabla \times B) \times \tilde{g})-\Lambda^{k}(\nabla \times B) \times \tilde{g}\right) d x,
\end{aligned}
$$

for we use the fact that $\int_{\mathbb{R}^{3}}\left(\nabla \times \Lambda^{k} B\right) \cdot\left(\left(\nabla \times \Lambda^{k} B\right) \times \tilde{g}\right) d x=0$. Using Lemma 2.1, we then derive that

$$
\begin{aligned}
\left|J_{2}\right| & \leq C \eta\|\nabla B\|_{H^{3}}\|\nabla B\|_{H^{2}}\|\tilde{g}\|_{W^{3, \infty}} \\
& \leq C \eta\|\nabla B\|_{H^{3}}\|B\|_{H^{3}}\|\tilde{g}\|_{W^{3, \infty}} \\
& \leq \frac{\mu}{16}\|\nabla B\|_{H^{3}}^{2}+C\|B\|_{H^{3}}^{2}\|\tilde{g}\|_{W^{3, \infty}}^{2} .
\end{aligned}
$$

For $J_{3}$, we could estimate it directly that

$$
\begin{aligned}
\left|J_{3}\right| & \leq C\|\tilde{g}\|_{W^{4, \infty}}\|B\|_{H^{3}}\|\nabla B\|_{H^{3}} \\
& \leq \frac{\mu}{16}\|\nabla B\|_{H^{3}}^{2}+C\|B\|_{H^{3}}^{2}\|\tilde{g}\|_{W^{4, \infty}}^{2} .
\end{aligned}
$$


For some quadratic $\nabla \Lambda^{3} B$ (highest derivatives) in $K_{1}, K_{2}$, we could not get good estimate of $K_{1}, K_{2}$ when $\tilde{g}$ is large. We also should not neglect $K_{0}, K_{3}$ containing some positive items. Therefore, we then find that it's a effective way to estimate $K_{1}, K_{2}, K_{0}$ and $K_{3}$ together.

$$
\begin{aligned}
& K_{0}+K_{1}+K_{2}+K_{3} \\
=- & \kappa \sum_{0 \leq k \leq 3} \int_{\mathbb{R}^{3}}\left|\left(\nabla \times \Lambda^{k} B\right) \times B\right|^{2}+2\left(\left(\nabla \times \Lambda^{k} B\right) \times B\right) \cdot\left(\tilde{g} \times\left(\nabla \times \Lambda^{k} B\right)\right) \\
& \quad+F_{0}+F_{1}+F_{2}+F_{3}-\left(\nabla \times \Lambda^{k} B\right) \times\left.\tilde{g}\right|^{2} d x \\
\leq & -\kappa \sum_{0 \leq k \leq 3} \int_{\mathbb{R}^{3}}\left(\left|\left(\nabla \times \Lambda^{\alpha} B\right) \times B\right|-\left|\left(\nabla \times \Lambda^{\alpha} B\right) \times \tilde{g}\right|\right)^{2}+F_{0}+F_{1}+F_{2}+F_{3},
\end{aligned}
$$

where

$$
\begin{aligned}
& F_{0}=\kappa \sum_{0 \leq k \leq 3} \sum_{|\beta| \leq 2, \beta+\gamma+\theta=k} \int_{\mathbb{R}^{3}}\left(\left(\left(\nabla \times \Lambda^{\beta} B\right) \times \Lambda^{\gamma} B\right) \times \Lambda^{\theta} B\right) \cdot\left(\nabla \times \Lambda^{k} B\right) d x, \\
& F_{1}=\kappa \sum_{0 \leq k \leq 3} \sum_{|\beta| \leq 2, \beta+\gamma+\theta=k} \int_{\mathbb{R}^{3}}\left(\left(\left(\nabla \times \Lambda^{\beta} B\right) \times \Lambda^{\gamma} B\right) \times \Lambda^{\theta} \tilde{g}\right) \cdot\left(\nabla \times \Lambda^{k} B\right) d x, \\
& F_{2}=\kappa \sum_{0 \leq k \leq 3} \sum_{|\beta| \leq 2, \beta+\gamma+\theta=k} \int_{\mathbb{R}^{3}}\left(\left(\left(\nabla \times \Lambda^{\beta} B\right) \times \Lambda^{\gamma} \tilde{g}\right) \times \Lambda^{\theta} B\right) \cdot\left(\nabla \times \Lambda^{k} B\right) d x, \\
& F_{3}=\kappa \sum_{0 \leq k \leq 3} \sum_{|\beta| \leq 2, \beta+\gamma+\theta=k} \int_{\mathbb{R}^{3}}\left(\left(\left(\nabla \times \Lambda^{\beta} B\right) \times \Lambda^{\gamma} \tilde{g}\right) \times \Lambda^{\theta} \tilde{g}\right) \cdot\left(\nabla \times \Lambda^{k} B\right) d x .
\end{aligned}
$$

For $F_{0}$, we have

$$
\begin{aligned}
F_{0} & \leq C\left(\|\nabla B\|_{H^{2}}\|\nabla B\|_{L^{\infty}}\|B\|_{L^{\infty}}+\|\nabla B\|_{L^{\infty}}\|\nabla B\|_{H^{2}}\|B\|_{L^{\infty}}+\|\nabla B\|_{L^{6}}^{3}\right)\|\nabla B\|_{H^{3}} \\
& \leq C\|\nabla B\|_{H^{3}}^{2}\|B\|_{H^{3}}^{2} .
\end{aligned}
$$

For $F_{1}$, we derive that

$$
\begin{aligned}
F_{1} & \leq C\|\tilde{g}\|_{W^{4, \infty}}\|B\|_{H^{3}}^{2}\|\nabla B\|_{H^{3}} \\
& \leq \frac{\mu}{16}\|\nabla B\|_{H^{3}}^{2}+C\|\tilde{g}\|_{W^{4, \infty}}^{2}\|B\|_{H^{3}}^{4} .
\end{aligned}
$$

For $F_{2}$, we could get

$$
\begin{aligned}
F_{2} \leq C\|\nabla B\|\left\|_{H^{2}}\left(\|\nabla B\|_{L^{\infty}}\|\tilde{g}\|_{L^{\infty}}+\|B\|_{L^{\infty}}\|\tilde{g}\|_{W^{1, \infty}}\right)\right\| \nabla B \|_{H^{3}} \\
\quad+C\|\nabla B\|_{W^{1,6}}\left(\|\nabla B\|_{W^{1,6}}\|\tilde{g}\|_{W^{1,6}}+\|B\|_{W^{1,6}}\|\tilde{g}\|_{W^{2,6}}\right)\|\nabla B\|_{H^{3}} \\
\leq \frac{\mu}{16}\|\nabla B\|_{H^{3}}^{2}+C\left(\|\tilde{g}\|_{W^{2, \infty}}^{2}+\|\tilde{g}\|_{W^{2,6}}^{2}\right)\|B\|_{H^{3}}^{4} .
\end{aligned}
$$

For $F_{3}$, it's easy for us th get

$$
\begin{gathered}
F_{3} \leq C|| \nabla B\left\|_{H^{2}}\right\| \tilde{g}\left\|_{L^{\infty}}^{2}\right\| \nabla B\left\|_{H^{3}}+\right\| \nabla B\left\|_{W^{1,6}}\right\| \tilde{g}\left\|_{W^{2,6}}^{2}\right\| \nabla B \|_{H^{3}} \\
\quad+C\|\nabla B\|_{L^{2}}\|\tilde{g}\|_{W^{3, \infty}}^{2}\|\nabla B\|_{H^{3}} \\
\leq \frac{\mu}{16}\|\nabla B\|_{H^{3}}^{2}+C\left(\|\tilde{g}\|_{W^{3, \infty}}^{4}+\|\tilde{g}\|_{W^{2,6}}^{4}\right)\|B\|_{H^{3}}^{2} .
\end{gathered}
$$


To estimate $K_{4}, K_{5}, K_{6}$, we have

$$
\begin{aligned}
K_{4} & \leq C\|\tilde{g}\|_{W^{4, \infty}}\|B\|_{H^{3}}\|\tilde{g}\|_{W^{3, \infty}}\|\nabla B\|_{H^{3}} \\
& \leq \frac{\mu}{16}\|\nabla B\|_{H^{3}}^{2}+C\|\tilde{g}\|_{W^{4, \infty}}^{4}\|B\|_{H^{3}}^{2}, \\
K_{5} & \leq C\|\tilde{g}\|_{W^{4, \infty}}\|B\|_{H^{3}}^{2}\|\nabla B\|_{H^{3}} \\
& \leq \frac{\mu}{16}\|\nabla B\|_{H^{3}}^{2}+C\|\tilde{g}\|_{W^{4, \infty}}^{2}\|B\|_{H^{3}}^{4}, \\
K_{6} \leq & C\|(\nabla \times \tilde{g}) \times \tilde{g}\|_{W^{3, \infty}}\|B\|_{H^{3}}\|\nabla B\|_{H^{3}} \\
\leq & \frac{\mu}{16}\|\nabla B\|_{H^{3}}^{2}+C\|\tilde{g}\|_{W^{4, \infty}}^{4}\|B\|_{H^{3}}^{2} .
\end{aligned}
$$

At last, we consider the term $\int_{\mathbb{R}^{3}} \Lambda^{k} G \Lambda^{k} B d x$. Recalling the expression of $G$, we have

$$
\begin{aligned}
\sum_{0 \leq k \leq 3}\left|\int_{\mathbb{R}^{3}} \Lambda^{k} G \Lambda^{k} B d x\right|= & \sum_{0 \leq} \mid \int_{\mathbb{R}^{3}} \Lambda^{k}\left(\nabla \times(\tilde{f} \times \tilde{g})+2 \nu \nabla \cdot\left(\nabla \chi_{M_{0}} g\right)-\nu \Delta \chi_{M_{0}} g\right. \\
& \left.+\frac{1}{2} f \cdot \nabla \chi_{M_{0}}^{2} g-\frac{1}{2} g \cdot \nabla \chi_{M_{0}}^{2} f\right) \cdot \Lambda^{k} B d x \mid \\
& \left.\left.+\sum_{0 \leq k \leq 3} \mid \int_{\mathbb{R}^{3}} \Lambda^{k}(\sigma(\nabla \times \tilde{g}) \times \tilde{g})-\kappa((\nabla \times \tilde{g}) \times \tilde{g}) \times \tilde{g}\right)\right) \cdot \Lambda^{k}(\nabla \times B) d x \mid \\
\leq & C|| \tilde{f} \times \tilde{g}\left\|_{H^{3}}\right\| \nabla B\left\|_{H^{3}}+\right\| \nabla \chi_{M_{0}} g\left\|_{H^{3}}\right\| \nabla B\left\|_{H^{3}}+C\right\| \Delta \chi_{M_{0}} g\left\|_{W^{3,6}}\right\| B \|_{W^{3,6}} \\
& +C\left(\left\|f \cdot \nabla \chi_{M_{0}}^{2} g\right\|_{W^{3, \frac{6}{5}}}+\left\|g \cdot \nabla \chi_{M_{0}}^{2} f\right\|_{W^{3, \frac{6}{5}}}\right)\|B\|_{W^{3,6}} \\
& +C\left(\|(\nabla \times \tilde{g}) \times \tilde{g}\|_{H^{3}}+C\|((\nabla \times \tilde{g}) \times \tilde{g}) \times \tilde{g}\|_{H^{3}}\right)\|\nabla B\|_{H^{3}} \\
\leq & C\left(\|\tilde{f} \times \tilde{g}\|_{H^{3}}+M_{0}^{-1}\|g\|_{H^{3}\left(|x| \leq 2 M_{0}\right)}\right)\|\nabla B\|_{H^{3}} \\
& +C M_{0}^{-2}\|g\|_{W^{3, \frac{6}{5}}\left(|x| \leq 2 M_{0}\right)}\|\nabla B\| H_{H^{3}} \\
& +C M_{0}^{-1}\|f \otimes g\|_{W^{3, \frac{6}{5}}\left(|x| \leq 2 M_{0}\right)}\|\nabla B\|_{H^{3}} \\
\leq & \left(\|\tilde{f} \times \tilde{g}\|_{H^{3}}+M_{0}^{-\frac{1}{2}} M_{2}\left|\alpha_{2}\right| e^{-\frac{\mu t}{4}}\right)\|\nabla B\|_{H^{3}} \\
& +C\left|\alpha_{1} \alpha_{2}\right| M_{0}^{-\frac{1}{2}} M_{2}^{2} e^{-\frac{(\mu+\nu) t}{4}}\|\nabla B\| H_{H^{3}} \\
& +C\left(\|(\nabla \times \tilde{g}) \times \tilde{g}\|_{H^{3}}+C\|((\nabla \times \tilde{g}) \times \tilde{g}) \times \tilde{g}\|_{H^{3}}\right)\|\nabla B\|_{H^{3}} .
\end{aligned}
$$

\section{Step 2: Energy inequalities of $U$.}

Operating Equation 3.1 with $\Lambda^{k}, 0 \leq k \leq 3$, and taking $L^{2}$ on Equation 3.1 yields

$$
\begin{aligned}
& \frac{1}{2} \frac{d}{d t}\|U\|_{H^{3}}^{2}+\mu\|\nabla U\|_{H^{3}}^{2} \\
= & H_{1}+H_{2}+H_{3}+H_{4}+H_{5}+H_{6} \\
& \quad+\sum_{0 \leq k \leq 3} \int_{\mathbb{R}^{3}} \Lambda^{k} F \Lambda^{k} U d x-\sum_{0 \leq k \leq 3} \int_{\mathbb{R}^{3}} \Lambda^{k} \nabla\left(p+\frac{1}{2}|\tilde{f}|^{2}-\frac{1}{2}|\tilde{g}|^{2}\right) \Lambda^{k} U d x,
\end{aligned}
$$


where

$$
\begin{aligned}
& H_{1}=-\sum_{0 \leq k \leq 3} \int_{\mathbb{R}^{3}} \Lambda^{k}(U \cdot \nabla U) \cdot \Lambda^{k} U d x, \quad H_{2}=-\sum_{0 \leq k \leq 3} \int_{\mathbb{R}^{3}} \Lambda^{k}(\tilde{f} \cdot \nabla U) \cdot \Lambda^{k} U d x, \\
& H_{3}=-\sum_{0 \leq k \leq 3} \int_{\mathbb{R}^{3}} \Lambda^{k}(U \cdot \nabla \tilde{f}) \cdot \Lambda^{k} U d x, \quad H_{4}=\sum_{0 \leq k \leq 3} \int_{\mathbb{R}^{3}} \Lambda^{k}(B \cdot \nabla B) \cdot \Lambda^{k} U d x, \\
& H_{5}=\sum_{0 \leq k \leq 3} \int_{\mathbb{R}^{3}} \Lambda^{k}(\tilde{g} \cdot \nabla B) \cdot \Lambda^{k} U d x, \quad H_{6}=\sum_{0 \leq k \leq 3} \int_{\mathbb{R}^{3}} \Lambda^{k}(B \cdot \nabla \tilde{g}) \cdot \Lambda^{k} U d x,
\end{aligned}
$$

Firstly, we have

$$
\begin{aligned}
& \quad\left|H_{1}+H_{2}\right| \\
& \leq \mid \sum_{0 \leq k \leq 3}\left(\int_{\mathbb{R}^{3}}\left(\Lambda^{k}(U \cdot \nabla U)-\left(U \cdot \nabla \Lambda^{k} U\right) \cdot \Lambda^{k} U d x+\int_{\mathbb{R}^{3}}\left(\Lambda^{k}\left(\tilde{f} \cdot \nabla U-\tilde{f} \cdot \nabla \Lambda^{k} U\right)\right) \cdot \Lambda^{k} U d x\right) \mid\right. \\
& \quad+\left|\sum_{0 \leq k \leq 3} \int_{\mathbb{R}^{3}}\left(\left(U \cdot \nabla \Lambda^{k} U\right) \cdot \Lambda^{k} U+\left(\tilde{f} \cdot \nabla \Lambda^{k} U\right) \cdot \Lambda^{k} U\right) d x\right| \\
& \leq \sum_{0 \leq k \leq 3} \mid\left(\int_{\mathbb{R}^{3}}\left(\Lambda^{k}(U \cdot \nabla U)-\left(U \cdot \nabla \Lambda^{k} U\right) \cdot \Lambda^{k} U d x+\int_{\mathbb{R}^{3}}\left(\Lambda^{k}\left(\tilde{f} \cdot \nabla U-\tilde{f} \cdot \nabla \Lambda^{k} U\right)\right) \cdot \Lambda^{k} U d x\right) \mid\right. \\
& \quad+\left|\sum_{0 \leq k \leq 3} \int_{\mathbb{R}^{3}}\left(u \cdot \nabla \Lambda^{k} U\right) \cdot \Lambda^{k} U d x\right| \\
& \leq \mid \sum_{0 \leq k \leq 3}\left(\int_{\mathbb{R}^{3}}\left(\Lambda^{k}(U \cdot \nabla U)-\left(U \cdot \nabla \Lambda^{k} U\right) \cdot \Lambda^{k} U d x|+| \int_{\mathbb{R}^{3}}\left(\Lambda^{k}\left(\tilde{f} \cdot \nabla U-\tilde{f} \cdot \nabla \Lambda^{k} U\right)\right) \cdot \Lambda^{k} U d x \mid\right)\right. \\
& \leq C \\
& \quad\left(\|\nabla U\|_{L^{\infty}}\|\nabla U\|_{H^{2}}+\|\nabla U\|_{L^{\frac{6}{\alpha}}}\|U\|_{\left.W^{3, \frac{6}{2-\alpha}}\right)}\right)\|U\|_{H^{3}} \\
& \quad+C\left(\|\nabla U\|_{H^{2}}\|\nabla \tilde{f}\|_{L^{\infty}}+\|\nabla U\|_{L^{6}}\|\tilde{f}\|_{W^{3,3}}\right)\|U\|_{H^{3}},
\end{aligned}
$$

By Sobolev inequality, we deduce that

$$
\left|H_{1}+H_{2}\right| \leq C\left(\left\|\left.\Lambda^{\frac{\alpha}{2}} U\right|_{H^{3}} ^{2}\right\| U\left\|_{H^{3}}+\right\| U \|_{H^{3}}^{2}\left(\|\tilde{f}\|_{W^{1, \infty}}+\|\tilde{f}\|_{W^{3,3}}\right)\right) .
$$

For $H_{3}$, it's easy for us to get

$$
\left|H_{3}\right| \leq\left. C|| U\right|_{H^{3}}\|B\|_{H^{3}} \mid \tilde{f} \|_{W^{4, \infty}} .
$$

Using the similar way to estimate $H_{1}+H_{2}$, we have

$$
\begin{aligned}
\left|H_{4}+H_{5}\right| \leq C & \left(\|\nabla B\|_{H^{2}}\|\nabla B\|_{L^{\infty}}+\|\nabla B\|_{L^{3}}\|B\|_{W^{3,6}}\right)\|U\|_{H^{3}} \\
& +C\left(\|\nabla B\|_{H^{2}}|| \nabla \tilde{g}\left\|_{L^{\infty}}+\right\| \nabla B\left\|_{L^{6}}\right\| \tilde{g} \|_{W^{3,3}}\right)\|U\|_{H^{3}} \\
\leq & C\left(\|\nabla B\|_{H^{3}}^{2}\|B\|_{H^{3}}+\|U\|_{H^{3}}\|B\|_{H^{3}}\left(\|\tilde{g}\|_{W^{1, \infty}}+\|\tilde{g}\|_{W^{3,3}}\right)\right)
\end{aligned}
$$

For $H_{6}$, we have

$$
\left|H_{6}\right| \leq C|| U\left\|_{H^{3}}\right\| B\left\|_{H^{3}}\right\| \tilde{g} \|_{W^{4, \infty}} .
$$


As for $\int_{\mathbb{R}^{3}} \Lambda^{k} F \Lambda^{k} U d x$, it suffices for us to have

$$
\begin{aligned}
\sum_{0 \leq k \leq 3}\left|\int_{\mathbb{R}^{3}} \Lambda^{k} F \Lambda^{k} U d x\right|= & \sum_{0 \leq k \leq 3} \mid \int_{\mathbb{R}^{3}} \Lambda^{k}\left(\tilde{f} \times(\nabla \times \tilde{f})-\tilde{g} \times(\nabla \times \tilde{g})-\nu \Delta \chi_{M_{0}} f\right. \\
& \left.+2 \nu \nabla \cdot\left(\nabla \chi_{M_{0}} f\right)\right) \cdot \Lambda^{k} U d x \mid \\
\leq & C\left(\|\tilde{f} \times(\nabla \times \tilde{f})\|_{H^{3}}+\|\tilde{g} \times(\nabla \times \tilde{g})\|_{H^{3}}\right)\|U\|_{H^{3}} \\
& +C\left(\left\|\nabla \chi_{M_{0}} f\right\|_{W^{4, \frac{6}{4+\alpha}}}+\left\|\Delta \chi_{M_{0}} f\right\|_{W^{3, \frac{6}{4+\alpha}}}\right)\|U\|_{W^{3, \frac{6}{2-\alpha}}} \\
\leq & C\left(\|\tilde{f} \times(\nabla \times \tilde{f})\|_{H^{3}}+\|\tilde{g} \times(\nabla \times \tilde{g})\|_{H^{3}}\right)\|U\|_{H^{3}} \\
& +C M_{0}^{-\frac{1}{2}} M_{2}\left|\alpha_{1}\right| e^{-\frac{\nu t}{2 \alpha}}\left\|\Lambda^{\frac{\alpha}{2}} U\right\|_{H^{3}} .
\end{aligned}
$$

We could estimate the pressure term in the same way with in [33]. Since

$$
\begin{aligned}
p= & (-\Delta)^{-1} \operatorname{div}(u \cdot \nabla u-b \cdot \nabla b) \\
= & \sum_{i, j}(-\Delta)^{-1} \partial_{i} \partial_{j}\left(u_{i} U_{j}-b_{i} B_{j}\right)+(-\Delta)^{-1} \nabla \cdot(U \cdot \nabla \tilde{f}-B \cdot \nabla \tilde{g}) \\
& \quad+(-\Delta)^{-1} \nabla \cdot(\tilde{f} \times(\nabla \times \tilde{f})-\tilde{g} \times(\nabla \times \tilde{g}))-\frac{1}{2}|\tilde{f}|^{2}+\frac{1}{2}|\tilde{g}|^{2},
\end{aligned}
$$

then we have

$$
\begin{aligned}
\Pi:=\mid & -\sum_{|k| \leq 3} \int_{\mathbb{R}^{3}} \Lambda^{k} \nabla\left(p+\frac{1}{2}|\tilde{f}|^{2}-\frac{1}{2}|\tilde{g}|^{2}\right) \Lambda^{k} U d x \mid \\
\leq & \left(\|u \otimes U\|_{W^{3, \frac{3}{2}}}+\|h \otimes B\|_{W^{3, \frac{3}{2}}}\right)\left\|f \cdot \nabla \chi_{M_{0}}\right\|_{W^{3,3}} \\
& +\|U \cdot \nabla \tilde{f}-B \cdot \nabla \tilde{g}\|_{H^{3}}|| U \|_{H^{3}} \\
& +\|\tilde{f} \times(\nabla \times \tilde{f})-\tilde{g} \times(\nabla \times \tilde{g})\|_{H^{3}}\|U\|_{H^{3}} .
\end{aligned}
$$

By Hölder's inequality, we furthermore derive that

$$
\begin{aligned}
\Pi \leq C & {\left[\left(\|U\|_{H^{3}}^{2}+\|\tilde{f}\|_{W^{3,6}}\|U\|_{H^{3}}\right)\left\|\nabla \chi_{M_{0}}\right\|_{W^{3, \infty}}\|f\|_{W^{3,3}\left(M_{0} \leq|x| \leq 2 M_{0}\right)}\right.} \\
& +\|H\|_{H^{3}}^{2} \cdot\left\|\nabla \chi_{M_{0}}\right\|_{W^{3, \infty}}\|f\|_{W^{3,3}\left(M_{0} \leq|x| \leq 2 M_{0}\right)} \\
& +\|\tilde{g}\|_{W^{3,2}}\|H\|_{W^{3,6}}\left\|\nabla \chi_{M_{0}}\right\|_{W^{3, \infty}}\|f\|_{W^{3,3}\left(M_{0} \leq|x| \leq 2 M_{0}\right)} \\
& +\left(\|\nabla \tilde{f}\|_{W^{3, \infty}}\|U\|_{H^{3}}+\|\nabla \tilde{g}\|_{W^{3, \infty}}\|B\|_{H^{3}}\right) \\
& +\left(\|\tilde{f} \times(\nabla \times \tilde{f})\|_{H^{3}}+\|\tilde{g} \times(\nabla \times \tilde{g})\|_{H^{3}}\right)\|U\|_{H^{3}} \\
& \left.+\|\nabla B\|_{H^{3}}\|(\nabla \times \tilde{g}) \times \tilde{g}\|_{H^{3}}+\|B\|_{H^{3}}^{2}\|\tilde{g}\|_{W^{5, \infty}}\right], \\
\leq & {\left[\left(\|U\|_{H^{3}}^{2}+\left|\alpha_{1}\right| M_{2} e^{-\frac{\mu t}{4}}\|U\|_{H^{3}}+\|B\|_{H^{3}}^{2} \alpha_{1} M_{2} M_{0}^{-1} e^{-\frac{\mu t}{4}}\right.\right.} \\
& +\left|\alpha_{2}\right| M_{2} M_{0}^{\frac{1}{2}} e^{-\frac{\nu t}{2^{\alpha}}}\|\nabla B\|_{H^{3}} \cdot \alpha_{1} M_{2} M_{0}^{-1} e^{-\frac{\mu t}{4}} \\
& +\left(\left|\alpha_{1}\right| M_{1} e^{-\frac{\mu t}{4}}\|U\|_{H^{3}}+\left|\alpha_{2}\right| M_{1} e^{-\frac{\nu t}{2^{\alpha}}}\|B\|_{H^{3}}\right)\|U\|_{H^{3}} \\
& \left.+\left(\alpha_{1}^{2} e^{-\frac{\nu t}{2^{\alpha-1}}}+\alpha_{2}^{2} e^{-\frac{\mu t}{2}}\right) \cdot\left(\delta M_{0}^{\frac{3}{2}} M_{1}^{2}+M_{0}^{-1} M_{2}^{2}\right)\|U\|_{H^{3}}\right] .
\end{aligned}
$$


In a result, we get

$$
\begin{aligned}
\Pi \leq C & \left(\left|\alpha_{1}\right|\left(M_{1}+M_{2}\right) e^{-\frac{\mu t}{4}}\|U\|_{H^{3}}^{2}+\left|\alpha_{1}\right| M_{2} e^{-\frac{\mu t}{4}}\|B\|_{H^{3}}^{2}\right) \\
& +C\left|\alpha_{2}\right| M_{1} e^{-\frac{\nu t}{2^{\alpha}}}\|U\|_{H^{3}}\|B\|_{H^{3}} \\
& +C\left(\alpha_{1}^{2} e^{-\frac{\nu t}{2^{\alpha}}}+\alpha_{2}^{2} e^{-\frac{\mu t}{2}}\right) \cdot\left(\delta M_{0}^{\frac{3}{2}} M_{1}^{2}+M_{0}^{-1} M_{2}^{2}\right)\|U\|_{H^{3}} \\
& +C\left|\alpha_{1} \alpha_{2}\right| M_{0}^{-\frac{1}{2}} M_{2}^{2} e^{-\frac{\left(2^{2-\alpha_{\nu+\mu) t}}\right.}{4}}\|\nabla B\|_{H^{3}} .
\end{aligned}
$$

\section{Step 3: Energy estimates of $U, B$.}

Gathering above estimates in Step 1 and Step 2, we obtain

$$
\frac{1}{2} \frac{d}{d t}\left(\|U\|_{H^{3}}^{2}+\|B\|_{H^{3}}^{2}\right)+\frac{\nu}{2}\|\nabla U\|_{H^{3}}^{2}+\frac{\mu}{2}\|\nabla B\|_{H^{3}}^{2}+P(t) \leq C \sum_{i=1}^{8} J_{i},
$$

where

$$
\begin{aligned}
& P(t)=\kappa \sum_{|k| \leq 3} \int_{\mathbb{R}^{3}}\left(\left|\left(\nabla \times \Lambda^{\alpha} B\right) \times B\right|-\left|\left(\nabla \times \Lambda^{\alpha} B\right) \times \tilde{g}\right|\right)^{2} d x, \\
& J_{1}=\left(\|U\|_{H^{3}}+\|B\|_{H^{3}}\right)\left(\|\nabla U\|_{H^{3}}^{2}+\|\nabla B\|_{H^{3}}^{2}\right) \text {, } \\
& J_{2}=\left(\|\tilde{f}\|_{W 4, \infty}+\|\tilde{g}\|_{W 4, \infty}\right)\left(\|U\|_{H^{3}}^{2}+\|B\|_{H^{3}}^{2}\right) \text {, } \\
& J_{3}=\left(\|\tilde{f} \times \tilde{g}\|_{H^{3}}+M_{0}^{-\frac{1}{2}} M_{2}\left|\alpha_{2}\right| e^{-\frac{\mu t}{4}}+\left|\alpha_{1} \alpha_{2}\right| M_{0}^{-\frac{1}{2}} M_{2}^{2} e^{-\frac{\left(2^{2-\alpha} \nu+\mu\right) t}{4}}\right)\|\nabla B\|_{H^{3}}, \\
& J_{4}=\left(|\sigma|||(\nabla \times \tilde{g}) \times \tilde{g}\left\|_{H^{3}}+\kappa||((\nabla \times \tilde{g}) \times \tilde{g}) \times \tilde{g}\right\|_{H^{3}}\right)\|\nabla B\|_{H^{3}} \\
& J_{5}=\left|\alpha_{1}\right|\left(M_{1}+M_{2}\right) e^{-\frac{\mu t}{4}}\|U\|_{H^{3}}^{2}+\|\tilde{g}\|_{W^{5, \infty}}\|B\|_{H^{3}}^{2}, \\
& J_{6}=\left|\alpha_{1}\right| M_{2} e^{-\frac{\mu t}{4}}\|B\|_{H^{3}}^{2}+\left|\alpha_{2}\right| M_{1} e^{-\frac{\nu t}{2^{\alpha}}}|| U\left\|_{H^{3}}\right\| B \|_{H^{3}}, \\
& J_{7}=\left(\alpha_{1}^{2} e^{-\frac{\nu t}{2^{\alpha-1}}}+\alpha_{2}^{2} e^{-\frac{\mu t}{2}}\right)\left(\delta M_{0}^{\frac{3}{2}} M_{1}^{2}+M_{0}^{-1} M_{2}^{2}\right)\|U\|_{H^{3}}, \\
& J_{8}=\left|\alpha_{1} \alpha_{2}\right| M_{0}^{-\frac{1}{2}} M_{2}^{2} e^{-\frac{\left(2^{2-\alpha} \nu+\mu\right) t}{4}}\|\nabla B\|_{H^{3}}, \\
& J_{9}=\left(\|\tilde{f} \times(\nabla \times \tilde{f})\|_{H^{3}}+\|\tilde{g} \times(\nabla \times \tilde{g})\|_{H^{3}}\right)\|U\|_{H^{3}}, \\
& J_{10}=\eta\|\nabla B\|_{H^{3}}\|B\|_{H^{3}}\|\tilde{g}\|_{W^{3, \infty}}+M_{0}^{-\frac{1}{2}} M_{2}\left|\alpha_{1}\right| e^{-\frac{\nu t}{2^{\alpha}}}\left\|\Lambda^{\frac{\alpha}{2}} U\right\|_{H^{3}}, \\
& J_{11}=C\|B\|_{H^{3}}^{4}\left(\|\tilde{g}\|_{W^{4, \infty}}^{2}+\|\tilde{g}\|_{W^{2,6}}^{2}\right)+C\|B\|_{H^{3}}^{2}\left(\|\tilde{g}\|_{W^{4, \infty}}^{4}+\|\tilde{g}\|_{W^{2,6}}^{4}\right) \text {. }
\end{aligned}
$$

Using Lemma 2.3. Young's inequality and $\delta^{-\frac{1}{2}} \geq M_{0} \gg 1$, we derive that

$$
\begin{aligned}
& \frac{d}{d t}\left(\|U\|_{H^{3}}^{2}+\|B\|_{H^{3}}^{2}\right)+\left(\frac{\nu}{2}-C\|U\|_{H^{3}}-C\|B\|_{H^{3}}\right)\left\|\Lambda^{\frac{\alpha}{2}} U\right\|_{H^{3}}^{2} \\
& \quad+\left(\frac{\mu}{2}-C\|U\|_{H^{3}}-C\|B\|_{H^{3}}\right)\|\nabla B\|_{H^{3}}^{2} \\
\leq C & \left(e^{-\frac{\nu t}{2^{\alpha}}}+e^{-\frac{\mu t}{4}}\right)\left(\|U\|_{H^{3}}^{2}+\|B\|_{H^{3}}^{2}+\|B\|_{H^{3}}^{4}\right)+C\left(M_{0}^{-1}+\delta^{2} M_{0}^{3}\right)\left(e^{-\frac{\nu t}{2 \alpha}}+e^{-\frac{\mu t}{4}}\right)
\end{aligned}
$$

for some constant $C$ depending on $M_{1}, M_{2}, \mu, \nu, \eta, \alpha_{1}, \alpha_{2}$. 
For $t \in[0, \infty)$, we assume that

$$
\|U(t)\|_{H^{3}}^{2}+\|B(t)\|_{H^{3}}^{2} \leq \frac{\min \{\mu, \nu\}}{4 C} .
$$

In case $t=0$, the above estimate holds. Applying differential inequality (3.31), Gronwall's inequality and $\delta \leq M_{0}^{-2}$, we have

$$
\|U(t)\|_{H^{3}}+\|B(t)\|_{H^{3}} \leq M_{0}^{-\frac{1}{2}} .
$$

In a result,

$$
\|U(t)\|_{H^{3}}+\|B(t)\|_{H^{3}} \leq M_{0}^{-\frac{1}{2}}
$$

for all $t \in[0, \infty)$. Therefore, we complete the proof of Theorem 1.1 .

\section{Acknowlegement}

The author is supported by Education Department of Hunan Province, general Program(grant No. 17C0039); the State Scholarship Fund of China Scholarship Council (No. 201808430121) and Hunan Provincial Key Laboratory of Intelligent Processing of Big Data on Transportation, Changsha University of Science and Technology, Changsha; 410114, China.

\section{References}

[1] M. Acheritogaray, P. Degond, A. Frouvelle and J.G. Liu: Kinetic formulation and global existence for the Hall-magneto-hydrodynamics system, Kinet. Relat. Models, 4 (2011), 901-918.

[2] D. Chae, P. Degond and J.G. Liu: Well-posedness for Hallmagnetohydrodynamics, Ann. I. H. Poincaré., 31 (2014), 555-565.

[3] L. Caffarelli and A. Vasseur: Drift diffusion equations with fractional diffusion and the quasi-geostrophic equation, Annals of Math., 171 (2010), 1903-1930.

[4] D. Chae and J. Lee: On the blow-up criterion and small data global existence for the Hall- magneto-hydrodynamics, J. Differential Equations, 26 (2014), 3835-3858.

[5] D. Chae and M. Schonbek: On the temporal decay for the Hall-magnetohydrodynamic equations, J. Differential Equations, 255 (2013), 3971-3982.

[6] D. Chae, R. Wan and J. Wu: Local well-posedness for the Hall-MHD equations with fractional magnetic diffusion, J. Math. Fluid Mech., 17 (2015), 627-638.

[7] D. Chae and S. Weng: Singularity formation for the incompressible Hall-MHD equations without resistivity, Ann. Inst. Henri Poincaré Anal. Non Linéaire, 33 (2016), 1009-1022.

[8] D. Chae and J. Wolf: On partial regularity for the 3D non-stationary Hall magnetohydrodynamics equations on the plane, SIAM J. Math. Anal., 48 (2016), 443-469. 
[9] J.Y. Chemin and I. Gallagher: Well-posedness and stability results for the Navier-Stokes equa tions in $R^{3}$, Ann. Inst. H. H. Poincaré Anal. Non Lineaire, 26 (2009), 599-624.

[10] P. Constantin and A. Majda: The Beltrami spectrum for incompressible fluid flows, Commun. Math. Phys., 115 (1988), 435-456.

[11] M.M. Dai: Regularity criterion for the 3D Hall-magneto-hydrodynamics. J. Differential Equations, 261 (2016), 573-591.

[12] P. A. Davidson: An Introduction to Magnetohydrodynamics, Cambridge University Press, Cambridge, 2001.

[13] N. Duan: Global well-posedness and analyticity of solutions to three-dimensional HallMHD equations, J. Math. Anal. Appl., 463 (2018) 506-516.

[14] J. Fan, X. Jia, G. Nakamura and Y. Zhou: On well-posedness and blowup criteria for the magnetohydrodynamics with the Hall and ion-slip effects, Z. Angew. Math. Phy., 66 (2015), 1695-1706.

[15] I.J. Jeong, S.J. Oh: On the Cauchy problem for the Hall and electron magnetohydrodynamic equations without resistivity I: illposedness near degenerate stationary solutions, arXiv:1902.02025v1

[16] S. Gala and M.A. Ragusa: On the blow-up criterion of strong solutions for the MHD equations with the Hall and ion-slip effects in $\mathbb{R}^{3}$, Z. Angew. Math. Phys., (2016) 67:18.

[17] Z. Lei, F.H. Lin and Y. Zhou: Structure of helicity and global solutions of incompressible Navier-Stokes equation, Arch. Ration. Mech. Anal., 218 (2015), 1417-1430.

[18] F.H. Lin, and P. Zhang: Global small solutions to an MHD-type system: the threedimensional case, Comm. Pure Appl. Math., 67 (2014), 531-580.

[19] Y.R. Lin, H.L. Zhang and Y. Zhou: Global smooth solutions of MHD equations with large data, J. Differential Equations, 261 (2016), 102-112.

[20] F. Liu and Y.Z Wang: Global solutions to three-dimensional generalized MHD equations with large initial data, Z. Angew. Math. Phys., (2019) 70:69.

[21] M. Kwak and B. Lkhagvasuren: Global wellposedness for Hall-MHD equations, Nonlinear Analysis, 174 (2018) 104-117.

[22] J.L. Li and X. Wu: Global smooth solutions of the 3D Hall-magnetohydrodynamic equations with large data. arXiv:1906.03953v1

[23] M. Maiellaro: Uniqueness of MHD thermodiffusive mixture flows with Hall and ion-slip effects. Meccanica 12, 9-14 (1977).

[24] G. Mulone and F. Salemi: Some continuous dependence theorems in MHD with Hall and ion-slip currents in unbounded domains. Rend. Ac. Sci. Fis. Mat. Napoli., 55 (1988), 139-152. 
[25] G. Mulone and V.A. Solonnikov: On an initial boundary-value problem for the equation of magnetohydrodynamics with the Hall and ion-slip effects. J. Math. Sci., 87(2), 33813392 (1997).

[26] W.M. Peng and Y. Zhou: Global large solutions to incompressible Navier-Stokes equations with gravity, Math. Meth. Appl. Sci., 38 (2015) 590-597.

[27] X.X. Ren, J.H Wu, Z.Y Xiang and Z.F. Zhang: Global existence and decay of smooth solution for the 2-D MHD equations without magnetic diffusion, J. Funct. Anal., 267 (2014), 503-541.

[28] M. Sermange and R. Temam: Some mathematical questions related to the MHD equations, Comm. Pure Appl. Math., 36 (1983), 635-664.

[29] E.M. Stein: Singular Integrals and Differentialbility Properties of Functions, Princeton University Press, Princeton, 1970.

[30] J. B. Taylor: Relaxation of Toroidal Plasma and Generation of Reverse Magnetic Fields, Phy. Rev. Letter, 33 (1974), 1138-1141.

[31] R. Wan: Global well-posedness to the 3D incompressible MHD equations with a new class of large initial data, arXiv:1509.07660v1

[32] K. Yamazaki and M. T. Moha: Well-posedness of Hall-magnetohydrodynamics system forced by Lévy noise, Stoch. PDE: Anal. Comp., (2018), 1-48.

[33] H.L. Zhang : Global large smooth solutions for 3-D Hall-magnetohydrodynamics, Discrete Cont. Dyn., 39 (2019), 6669-6682.

[34] X.P. Zhao and M.X. Zhu: Global well-posedness and asymptotic behavior of solutions for the three-dimensional MHD equations with Hall and ion-slip effects. Z. Angew. Math. Phys. 69 (2018), no. 2, Art. 22, 13 pp.

[35] Y. Zhou and Y. Zhu : A class of large solutions to the 3D incompressible MHD and Euler equations with damping, Acta Math. Sinica English Series, 34 (2018), 63-78. 